\title{
Operational reliability evaluation of PV inverter considering relative humidity and its application on power system
}

\author{
Yangyang Tan*, Jun Liu, Sichang Xu, Peng Zhong, Qi Zhang and Lin Hu \\ State Grid Chengdu Power Supply Company, Chengdu, Sichuan, 610041, China
}

\begin{abstract}
With the penetration of renewable energy in the power system gradually increases, the importance of power electronics is growing up. The reliability of the power electronics should be taken seriously. This paper focuses on the operational reliability of photovoltaic (PV) inverters which is the most vulnerable in grid-connected PV systems and its application on the reliability evaluation of power systems. According to the field data, the effect of relative humidity is nonnegligible to the reliability of PV inverters. First, the real-time failure rate of components in PV inverters calculation method considering relative humidity is presented. Then the operational reliability evaluation of PV inverters is proposed. Finally, the reliability of power system including grid-connected PV systems is evaluated. It is aimed to explore a bottom-up approach to "effect factors-components-devices-system" reliability evaluation to establish a link between the components/devices and system. In this paper, the numerical examples verify the necessary of considering relative humidity in reliability evaluation. The evaluation results of PV inverters are used to the Roy Billinton Test System (RBTS). The analysis shows the results may affect the overall system performance.
\end{abstract}

\section{Introduction}

The power electronics are the most prone to failure in the power system. The reason is that power electronics often withstand high voltage, high current and extremely high switching frequency when they operate in the power system. Therefore, the reliability of power electronics is the foundation and guarantee for the reliable operation of power electronic-based system. And it will affect the reliability of power systems.

In the power systems, existing reliability studies are generally divided into two categories: component/device level and system level. The former is mainly for power components or power devices. These include: one is theoretical life calculation based on mission profile and classic life model [1-3], mostly in the laboratory for simulation. However, it is greatly different between the simulation results and the actual operational results. Another is fault statistics or reliability studies based on field data usually focus on power devices [4-5]. However, this type of studies requires a large amount of long-term data collection and statistics, and it has a strong regional specificity. The latter is the reliability of power systems. For the traditional system reliability is based on probability and mathematical statistics and the parameters of reliability especially the failure rate is constant [6-8]. Based on the reliability of traditional power system, the operational reliability is proposed, the parameters of reliability for elements are variable.
However, few studies have established a link between the two categories.

In fact, it is hard to simulate all operational conditions because of the restricted laboratory conditions and the large number of operational conditions. The literature [4] has carried out an analysis to identify the relative humidity is one of the root causes and driving factors of faults in MOSFETs. However, the relative humidity is not considered into the operational reliability evaluation of photovoltaic (PV) inverters. Moreover, with the change of operation conditions, the reliability parameters of power system will also change. So, the effect of operational reliability of PV inverters should be taken account into the reliability of power systems.

This paper tends to establish a bottom-up approach to the "effect factors-components-devices-system" reliability evaluation for establishing a link between the components/devices and system. First, establish the relationship between effect factors and real-time failure rate of components in PV inverters. Then, the operational reliability of PV inverters is evaluated according to the topology of PV inverters. Finally, the reliability of power system is evaluated considering the operational reliability of PV inverters. In the numerical example, the necessity of considering the relative humidity is proved, and the application of the operational reliability of PV inverters on the power system is analysed in the Roy Billinton Test System (RBTS). 


\section{Effect of relative humidity on PV inverters}

\subsection{Failure Rate Calculation of Power Electronic Components}

According to the FIDES Guide 2009 [9], the failure rate of semiconductors can be calculated by

$$
\lambda_{\text {sem }}=\lambda_{\text {physical }} \times \prod_{P M} \times \prod_{P R}
$$

Where, $\lambda_{\text {sem }}$ is the failure rate of components. $\lambda_{\text {physical }}$ represents the physical contribution. $\Pi_{P M}$ represents the quality and technical control over manufacturing of the components. $\Pi_{P R}$ represents the quality and technical control over the development, manufacturing and usage process for the product containing the components.

Physical stress refers to all the stresses that the component bears in the operation, including the variations of external environment and operation conditions. This paper focuses on the ambient environmental factors, including solar radiation intensity, temperature and relative humidity. The physical contribution factor is calculated by

$$
\lambda_{\text {physical }}=\left(\lambda_{\text {OTH }} \times \prod_{T H}+\lambda_{0 T C} \times \prod_{T C}+\lambda_{0 R H} \times \prod_{R H}\right) \times \prod_{I N}
$$

Where, $\Pi_{I N}$ represents the contribution of induced factors (also called over stresses) inherent to an application field. $\Pi_{T H}$ represents the thermal and electrical factor. $\Pi_{T C}$ represents the temperature cycling factor. $\Pi_{R H}$ represents the humidity factor. $\lambda_{0}$ represents the basic failure rates for the different physical stresses. Different items correspond to different constants.

\section{- Thermal and electrical factor}

The thermal and electrical factor only effects during on-state periods of power electronic components.

$$
\prod_{T H}=e^{11604 \times 0.44 \times\left[\frac{1}{293}-\frac{1}{T_{a m}+\Delta T+273}\right]}
$$

Where, $T_{a m}$ is average temperature of the component.

- Temperature cycling factor



Where, $t_{\text {annual }}$ is time associated with each phase over a year (hours). $N_{\text {annual-cycling }}$ is number of cycles associated with each cycling phase over a year (cycles). $\theta_{c y}$ is cycle duration (hours). $\Delta T_{\text {cycling }}$ is amplitude of variation associated with a cycling phase $\left({ }^{\circ} \mathrm{C}\right) . T_{\text {cycling-max }}$ is maximum temperature of the sub-assembly during a cycling phase $\left({ }^{\circ} \mathrm{C}\right)$.

- Relative humidity factor

The relative humidity factor only effects during offstate period of power electronic components.

$$
\prod_{R H}=\left(\frac{R H}{70}\right)^{4.4} \times e^{11604 \times 0.6 \times\left[\frac{1}{293}-\frac{1}{\left(T_{a m}+273\right)}\right]}
$$

Where, $R H$ is relative humidity associated with a phase $(\%)$

\subsection{Losses of Power Electronic Components Calculation}

- The switching losses of components

$$
\begin{gathered}
P_{s w, T}=\frac{1}{\pi} I_{m} f_{s}\left(\Delta E_{O N}+\Delta E_{O F F}\right) \\
P_{s w, \mathrm{D}}=\frac{1}{\pi} I_{m} f_{s}\left(\Delta E_{r r}\right)
\end{gathered}
$$

Where, $P_{s w, T}$ is the conduction losses of the IGBT, $P_{s w, D}$ is the conduction losses of the diode. $\Delta E_{r r}$ is the rated switching energy loss of the diode, $\Delta E_{\text {on }}, \Delta E_{\text {off }}$ are the energy losses in the ON and OFF state, respectively. $f_{s w}$ is the switching frequency.

- The conduction losses of components

$$
P_{c o n, T}=\frac{1}{8} R_{T} I_{m}^{2}+\frac{1}{2 \pi} V_{C E 0} I_{m}+\frac{M}{3 \pi} R_{T} I_{m}^{2} \cos \varphi+\frac{M}{8} V_{C E 0} I_{m} \cos \varphi
$$

$$
P_{c o n, D}=\frac{1}{8} R_{D} I_{m}^{2}+\frac{1}{2 \pi} V_{F 0} I_{m}-\frac{M}{3 \pi} R_{D} I_{m}^{2} \cos \varphi-\frac{M}{8} V_{F 0} I_{m} \cos \varphi
$$

Where, $P_{c o n, T}$ is the conduction losses of the IGBT, $P_{c o n, D}$ is the conduction losses of the diode. $V_{C E 0}, V_{F 0}$ are the voltage drops on the IGBT and diode, respectively. M is modulation coefficient. $\cos \varphi$ is power factor. $I_{m}$ is the peak of phase current. $R_{T}, R_{D}$ are the resistances of IGBT and diode, respectively.

- The temperature of components

The ambient temperature of the component can be expressed by junction temperature, it can be calculated by

$$
T_{j}=T_{a}+P_{D / T} R_{D / T}
$$

Where, $T_{a}$ is the ambient temperature in which the component operates. $R_{T}, R_{D}$ are the thermal resistance of IGBT and diode, respectively. $P_{T}, P_{D}$ are the power loss of IGBT and diode, respectively.

- Calculating the peak of phase current

$$
I_{m} \approx \frac{P_{i n}(t)}{\sqrt{2} V_{d}}
$$

Where, $P_{\text {in }}(\mathrm{t})$ is the generation of Grid-connected PV. $V_{d}$ is the dc input voltage. 


\section{The operational reliability evaluation of PV inverter}

\subsection{Failure Rate Calculation of PV Inverter}

The failure rate calculation of PV inverters will be different when the topology is different. A typical singlephase inverter consists of a connection of IGBTs and diodes, as shown in Figure 1.



Figure 1. Single-phase full-bridge inverter topology.

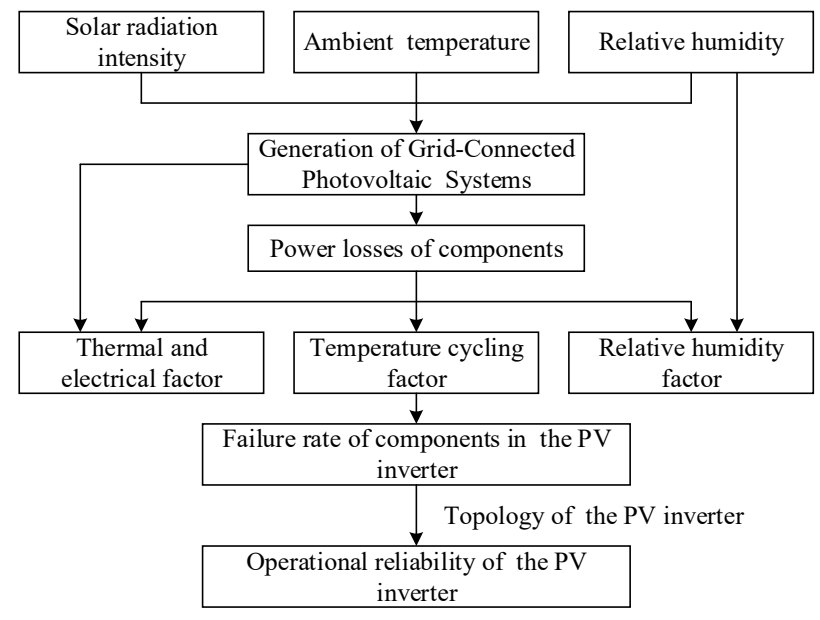

Figure 2. Flow chart of operational reliability evaluation for $\mathrm{PV}$ inverters

The failure rate of $\mathrm{PV}$ inverter is

$$
\lambda_{i n v}=3 \times\left(2 \times \lambda_{T}+2 \times \lambda_{D}\right)
$$

Where, $\lambda_{i n v}$ represents the failure rates of high-power photovoltaic inverter. $\lambda_{T}$ represents the failure rates of the IGBT. $\lambda_{D}$ represents the failure rates of the diode.

\subsection{The Operational Reliability Evaluation of PV Inverter}

The operational reliability of PV inverters can be described by the real-time failure rate of PV inverters. The real-time failure rate is calculated by

$$
\lambda_{i n v}(\mathrm{t})=\sum_{t_{i}}^{T}\left(\frac{t_{i}}{8760}\right)\left(\lambda_{i n v}\right)_{t_{i}}
$$

Where, $t_{i}$ is current period, $T$ is overall running time, $\left(\lambda_{\text {inv }}\right)_{\mathrm{ti}}$ is the failure rate of current period.
Based on the aforementioned analysis, the flow chart of operational reliability evaluation of PV inverters considering the effect of relative humidity is shown in Figure 2.

\section{Application on the reliability of power system}

The reliability of power system is the ability to provide uninterrupted power to customers. For the power system with grid-connected PV systems, once the PV inverters are in outage, the reliability of power system will be affected. So, it should be considered the operational reliability of PV inverters to the reliability evaluation of power electronic-based system.

\subsection{Availability of PV inverter}

a run-to-fail replacement strategy is employed for availability prediction in this paper. The availability of $\mathrm{PV}$ inverters can be calculated by

$$
A_{i n v}=\frac{\mu_{i n v}}{\lambda_{i n v}+\mu_{i n v}}
$$

Where, $\mu_{i n v}$ is the repair rates of PV inverters.

\subsection{Reliability Evaluaition of Power System}

Reliability evaluation of power system is measured by indices such as loss of load probability (LOLP) and expected energy not supplied (EENS).

loss of load probability (LOLP) is the probability within a specific period in which the load cannot be met due to the generation shortage, and it can be calculated by

$$
L O L P=\sum_{i \in S} P_{i}
$$

Where, $S$ is the set of system states that can't meet the load within a specific phase. $P_{i}$ is the probability corresponding to the system state $i$ that can't meet the load.

EENS is also defined as the curtailed energy due to the generation shortage and it is calculated by

$$
E E N S=\sum_{i \in S} P_{i} E_{i}
$$

Where, $E_{i}$ is the curtailed energy.

Table 1. Parameters of components

\begin{tabular}{cc||cc}
\hline \multicolumn{2}{c||}{ Diode } & \multicolumn{2}{c}{ IGBT } \\
\cline { 3 - 4 } Parameters & Value & Parameters & Value \\
\hline $\mathrm{V}_{\mathrm{F} 0}$ & $1.85 \mathrm{~V}$ & $\mathrm{~V}_{\text {ceo }}$ & $2.05 \mathrm{~V}$ \\
$\mathrm{R}_{\mathrm{D}}$ & $0.652 \mathrm{~m} \Omega$ & $\mathrm{R}_{\mathrm{T}}$ & $0.816 \mathrm{~m} \Omega$ \\
$\Delta \mathrm{E}_{\text {rr }}$ & $0.0786 \mathrm{~mJ} / \mathrm{A}$ & $\Delta \mathrm{E}_{\text {on }}$ & $0.0571 \mathrm{~mJ} / \mathrm{A}$ \\
$\Delta \mathrm{E}_{\text {on }} / \Delta \mathrm{E}_{\text {off }}$ & - & $\Delta \mathrm{E}_{\text {off }}$ & $0.02 \mathrm{~mJ} / \mathrm{A}$ \\
\hline
\end{tabular}


Table 2. Parameters of PV Inverter

\begin{tabular}{c|c}
\hline Parameters & Value \\
\hline Rated power $(\mathrm{MW})$ & 50 \\
\hline $\mathrm{M}$ & 0.9 \\
\hline switching frequency $(\mathrm{kHz})$ & 2.5 \\
\hline
\end{tabular}

\section{Numerical examples}

The reliability of a typical single-phase inverter consists of a connection of IGBTs and diodes, as shown in Figure



Figure 3. Solar radiation intensity.



Figure 5. Relative humidity.



Figure 7. Effect of value of relative humidity on operational reliability of PV inverters.

\subsection{Effect of Relative Humidity}

In order to analyse the effect of relative humidity on the operational reliability of PV inverters, the real-time
1, is evaluated the operational reliability using the procedure shown in Figure 2.

The parameters of the PV inverters are presented in Tables 1 and 2, respectively. In order to focus on the effect of relative humidity on reliability of PV inverters, the other components except IGBTs and Diodes are considered completely reliable.

The actual solar radiation intensity, ambient temperature and relative humidity time series in Xinjiang Province, China, which are shown in Figure 3.-Figure 5., respectively, are used in the examples.



Figure 4. Ambient temperature.



Figure 6. Effect of relative humidity on operational reliability of PV inverters.



Figure 8. Effect of off-state periods on operational reliability of PV inverters.

failure rate of PV inverters is calculated using the field data in Xinjiang Province, China in 2018. Then the realtime failure rate of PV inverters is calculated without 
relative humidity. It can be seen from Figure6 that the results are of difference.

It can be obtained from Figure 6, operational reliability of PV inverters is obviously influenced by the relative humidity. Therefore, the effect of relative humidity on the operational reliability of PV inverters is nonnegligible.

As mentioned earlier, the effect of relative humidity on operational reliability of PV inverters is divided into two parts: value of relative humidity and off-state periods. Then, the sensitivity of value of relative humidity and off-state periods will be presented.



Figure 9. Topology of modified RBTS.

\section{- Off-state periods}

Comparing the real-time failure rate of PV inverters at different off-state periods, the results is shown as Figure 8 .

It is interesting from the Fig. 8 that the real-time failure rate of PV inverters varies slowly when off-state periods are more than 3800 hours. That is to say, the operational reliability of PV inverters is sensitive to offstate periods only when off-state periods are less than 3800 hours.

\subsection{Application on Reliability of Power System}

The test system is the modified RBTS with additional 50 MW grid-connected PV system with $17 \%$ penetration. The structure of the modified RBTS is shown in Figure 9. The reliability of modified RBTS by LOLP and EENS indices to analysis the effect of reliability of PV inverters.

The impact of operational reliability of PV inverters on the system reliability is illustrated in Figure 10 and Figure 11. The real-time failure rate of PV inverters is

\section{- Value of relative humidity}

Assuming that relative humidity of the whole year is in different level, the operational reliability evaluation results of PV inverters are shown as Figure 8.

As we can see from Figure 7 that with relative humidity increases, the real-time failure rate of PV inverters is more sensitive. That is to say, the relative humidity is higher, the effect of relative humidity on the operational reliability of PV inverters is greater. When the PV inverters in the high relative humidity condition, the reliability of PV inverters should be closely attention.



Figure 10. LOLP of modified RBTS

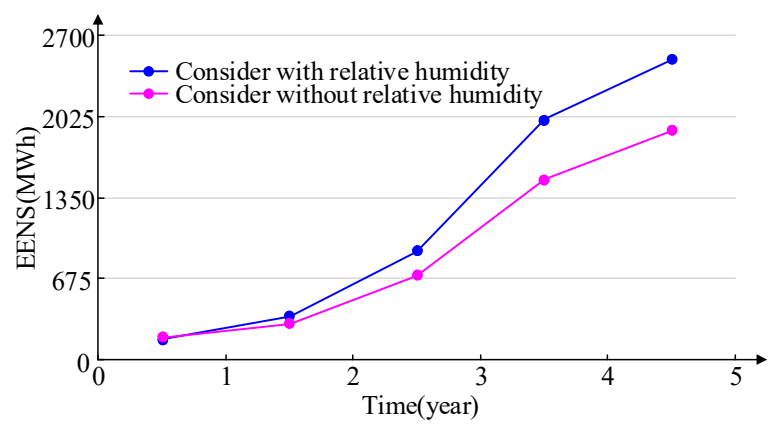

Figure 11. EENS of modified RBTS

considered. The obtained results show that the difference of effect on the operational reliability of PV inverters on the LOLP is $2.8 \%$ to $30 \%$ and EENS is $9.6 \%$ to $30 \%$. Therefore, for the reliability of power system, the effect of operational reliability of PV inverters can't be neglected.

\section{Conclusion}

This paper investigated the effect of relative humidity on operational reliability evaluation of PV inverters and the effect of operational reliability evaluation of PV inverters on the reliability of power system. The numerical examples has been proved the effect of relative humidity on operational reliability evaluation of PV inverters is nonnegligible. What's more, the effect of operational reliability of PV inverters can't be neglected to the reliability of power system.

This paper explores a bottom-up approach to "relative humidity-components-PV inverters- power system" reliability evaluation. It provides a reference for more accurately evaluating the reliability of the power system 
in combination with comprehensive factors such as the health of devices, environmental impact, and system operational conditions. It is the future research to consider more influencing factors on field condition and extend this method to more power system devices, then form a complete theoretical framework.

\section{References}

1. Shen, Y. F., Wang, H., Blaabjerg, F. (2017) Reliability oriented design of a grid-connected photovoltaic microinverter. In: IEEE 3rd International Future Energy Electronics Conference and ECCE Asia. Kaohsiung. pp. 81-86.

2. Gu, B., Dominic, J., Lai, J., Chen, C., LaBella, T., Chen, B. (2013) High reliability and efficiency single-phase transformerless inverter for gridconnected photovoltaic systems. IEEE Transactions on Power Electronics, 28: 2235-2245.

3. Zhang, P., Wang, Y., Xiao, W., Li, W. (2012) Reliability Evaluation of Grid-Connected Photovoltaic Power Systems. IEEE Transactions on Sustainable Energy, 3: 379-389.

4. Sadik, D., Nee, H., Giezendanner, F., Ranstad, P. (2016) Humidity testing of SiC power MOSFETs. In: IEEE 8th International Power Electronics and Motion Control Conference. Hefei. pp. 3131-3136.

5. Yang, S., Bryant, A., Mawby, P., Xiang, D., Ran, L. Tavner, P. (2011) An industry-based survey of reliability in power electronic converters. IEEE Transactions on Industry Applications, 47: 14411451.

6. Billinton, R. (1969) Composite system reliability evaluation. IEEE Transactions on Power Apparatus and Systems, PAS-88: 276-281.

7. Xie, K., Hu, B., Singh, C. (2016) Reliability evaluation of double 12-pulse ultra HVDC transmission systems. IEEE Transactions on Power Delivery, 31: 210-218.

8. Cadini, F., Agliardi, G., Zio, E. (2017) A modeling and simulation framework for the reliability/availability assessment of a power transmission grid subject to cascading failures under extreme weather conditions. Applied Energy, 185: 267-279.

9. FIDES. (2009) FIDES Guide 2009 Edition A. https://www.fides-reliability.org/en/node/610. 